\title{
Syphilis reinfection is associated with an attenuated immune profile in the same individual: a prospective observational cohort study
}

\author{
Chris Kenyon ${ }^{1,2^{*}}$, Kara Krista Osbak', Tania Crucitti ${ }^{3}$ and Luc Kestens ${ }^{4,5}$
}

\begin{abstract}
Background: Ascertaining if the clinical and immunological response to repeat syphilis differs from that in initial syphilis may assist in designing optimal syphilis screening strategies and vaccine design.

Methods: We prospectively recruited 120 patients with a new diagnosis of (baseline) syphilis. During a 24-month follow-up period, 11 of these patients had a further diagnosis of (repeat) syphilis. We conducted a paired comparison of their plasma cyto-chemokines at baseline and repeat syphilis.

Results: Comparing to their baseline infection, paired analyses of the 11 individuals with repeat infections during follow-up revealed that these reinfections had lower concentrations of Interferon (IFN)a (0.8 [Interquartile range (IQR) $0.8-0.8$ vs. 12.2 [IQR 1.6-24.2], $P=0.004$ ) and Chemokine (C-C motif) ligand (CCL) 4 (0.9 [IQR 0.9-12.2 vs. 17.5 [IQR 4.9-32.8], $P=0.022]$.

Conclusion: In this small study of 11 individuals, repeat syphilis was found to present with an attenuated immune response. The relevance of these findings to the design of optimal syphilis screening programs is discussed.
\end{abstract}

Keywords: Syphilis, Immunology, Repeat, Screening, Cytokine

\section{Background}

A striking feature of the current epidemics of syphilis in high-income countries is the increasing proportion of cases of syphilis that are repeat episodes of syphilis [1-3]. These infections occur predominantly in men who have sex with men (MSM) with high rates of partner change [1, 4]. There is growing recognition that part of the difficulty with controlling these epidemics is the fact that repeat syphilis is less likely to present with symptoms [4-8]. As a result, persons with repeat syphilis may have delayed diagnoses and be more likely to transmit syphilis $[1,6]$. At our medical centre in Antwerp, Belgium, reinfections now constitute approximately half of all syphilis infections [1]. In a study that followed up 120 patients with a new diagnosis of syphilis over a 12-month period we previously confirmed that repeat syphilis was not only less likely to be symptomatic than initial syphilis but also had an altered immune profile [4]. These differences included variations in the magnitude and decay curves of rapid plasma reagin (RPR) as well as repeaters being less likely to have elevated plasma interleukin (IL)-10. These differences in plasma IL-10 persisted at 6-month follow-up such that those with initial but not those with repeat syphilis had higher levels of IL-10 than the control group. While following up this cohort for a 24-month period a total of 11 individuals had a further repeat episode of syphilis. This manuscript compares the immune responses of these 11 repeat episodes with the paired baseline episodes.

\footnotetext{
* Correspondence: ckenyon@itg.be

${ }^{1} \mathrm{HIV} / \mathrm{STI}$ Unit, Institute of Tropical Medicine, Antwerp, Belgium

${ }^{2}$ Division of Infectious Diseases and HIV Medicine, University of Cape Town,

Anzio Road, Observatory, Cape Town 7700, South Africa

Full list of author information is available at the end of the article
}

\section{Methods}

The Institutional Review Board of the Institute of Tropical Medicine and the Ethics Committee of the University Hospital Antwerp approved this study (13/44/426). All 
study participants provided written informed consent. Between May 2014 and June 2015 all patients attending the Institute of Tropical Medicine, Antwerp's STI or HIV clinics and over the age of 17 years in whom a new diagnosis of syphilis was made were prospectively recruited into the study (ClinicalTrials.gov Registration Number: NCT02059525). The diagnosis and staging of syphilis was according to the Centers for Disease Control and Prevention classification [9]. Repeat syphilis, which included syphilis repeat infections and reactivations, was defined as an episode in a person who had $\mathrm{a} \geq 4$ increase in RPR titre after a previous diagnosis of syphilis who exhibited an appropriate response to therapy, defined as $\mathrm{a} \geq 4$-fold decrease in RPR titre. All patients were assessed according to a standardized protocol that collected detailed information about sexual behavior, clinical features and laboratory tests. Patients were followed up at 3, 6, 9, 12, 18, 24 months and at any other time if they developed symptoms or signs of syphilis. Patient clinical and laboratory characteristics were recorded at each consultation. Blood was drawn into plain and EDTA-coated tubes (Sarstedt Monovette, Nümbrecht, Germany) and centrifuged at $2000 \mathrm{~g}$ for $10 \mathrm{~min}$ at ambient temperature. Serum and plasma was stored at $-20{ }^{\circ} \mathrm{C}$ (plain) and $-80^{\circ} \mathrm{C}$ (plasma), respectively. Samples were processed and frozen within $3 \mathrm{~h}$ of acquisition.

Interferon (IFN) $\alpha$, IFN $\gamma$, IL-1 $\beta$, IL-12p40, IL-12p70, Interferon gamma inducible protein (IP)-10, Monocyte chemoattractant protein (MCP)-1, Macrophage inflammatory protein (MIP)-1 $\alpha / /$ Chemokine (C-C motif) ligand (CCL)3, CCL4 (MIP-1ß), IL-4, IL-5, IL-6, IL-7, IL-8, IL-10 and IL-17A from the baseline, 6 month follow-up and repeat episodes of syphilis were measured in a single experiment using a magnetic bead Luminex multiplex assay (EMD Millipore, Billerica, MA, United States (US)) according to the manufacturer's instructions. Samples below the limit of quantification were assigned the value of half the lowest limit detected for each cytokine (for further study details including quality assessment data please see [4]). Macro Vue RPR card test (Becton, Dickinson and Company, Sparks, MD, US) and Treponema pallidum agglutination chemiluminescent immunoassay (Ortho-Clinical Diagnostics, Rochester, NY, US) performed on the Vitros 5600 Integrated System (Ortho-Clinical Diagnostics) were performed at each study visit.

\section{T. pallidum $\mathrm{PCR}$}

An in-house real time PCR targeting polA was used to detect T. pallidum DNA in serum. DNA was extracted from $400 \mu \mathrm{L}$ serum using the custom-plasma program of the Abbott m2000sp automated extraction platform (Abbott, Maidenhead, UK), according to the manufacturer's specifications. The extracted DNA was eluated in $100 \mu \mathrm{L}$ eluate solution. The $25 \mu \mathrm{L}$ PCR mixture contained Platinum ${ }^{\circ}$ Quantitative PCR SuperMix-Uracil DNA Glycosylase (Invitrogen by life technologies, Thermo Fisher Scientific) $0.9 \mu \mathrm{M}$ forward primer TP-1, $0.9 \mu \mathrm{M}$ reverse primer TP-2,0.14 $\mu \mathrm{M}$ probe TP-3 (Integrated DNA technologies, Leuven, Belgium) [10] and $10 \mu \mathrm{L}$ DNA extract. The amplifications were performed using the Rotor Gene 6000 (Qiagen, Venlo, the Netherlands) and included 45 cycles of $20 \mathrm{~s}$ at $95{ }^{\circ} \mathrm{C}$ followed by $45 \mathrm{~s}$ at $60{ }^{\circ} \mathrm{C}$ per cycle.

\section{Statistical analysis}

Values are summarized as medians and interquartile ranges (IQR). Wilcoxon's signed rank test was used to evaluate intraindividual changes from baseline to repeat syphilis. Spearman's correlation was used to assess if there was an association between the number of prior episodes of syphilis and plasma cytokine and chemokine concentrations at the time of syphilis diagnosis (baseline and repeat infections combined, $N=22$ ). All analyses were performed in Stata 13 (StataCorp LP, College Station, TX, USA). Due to the small sample size we did not conduct multivariable analyses.

\section{Results}

The cohort was followed up for 24 months. During this time period 11 individuals (all men, MSM and 10 HIV-infected) were diagnosed with a syphilis reinfection a median 456 (IQR 364-503) days after their baseline infection (Table 1). The median CD4+ T-cell count of the 10 HIV-infected individuals was 554/ $\mu \mathrm{L}$ (IQR 489-677) at the baseline visit. All HIV-infected individuals were on antiretroviral therapy with undetectable HIV viral loads. Serum PCR for Treponema pallidum was negative for all 11 individuals at baseline and for $6 / 6$ at the time of reinfection. All 11 were treated with stage appropriate intramuscular benzyl penicillin G (BPG) therapy at baseline and reinfection syphilis.

\section{Syphilis stage at reinfection}

Two of these reinfections were primary, two secondary and seven early latent syphilis (Table 1).

\section{Only one individual presented with confirmed initial syphilis}

For 10 of these individuals, this represented at least their third episode of syphilis (Table 1). The 11th individual was the only one to present with an initial episode of syphilis. His first episode of syphilis was characterized by a diffuse maculopapular rash and a RPR titre of $1 / 32$. He had tested TPA and RPR negative 6 months prior. He received 2.4MU BPG via intramuscular injection and his RPR titre declined to $1 / 4$ at 3 months. At nine months following condomless sex, he presented with a less pronounced maculopapular 


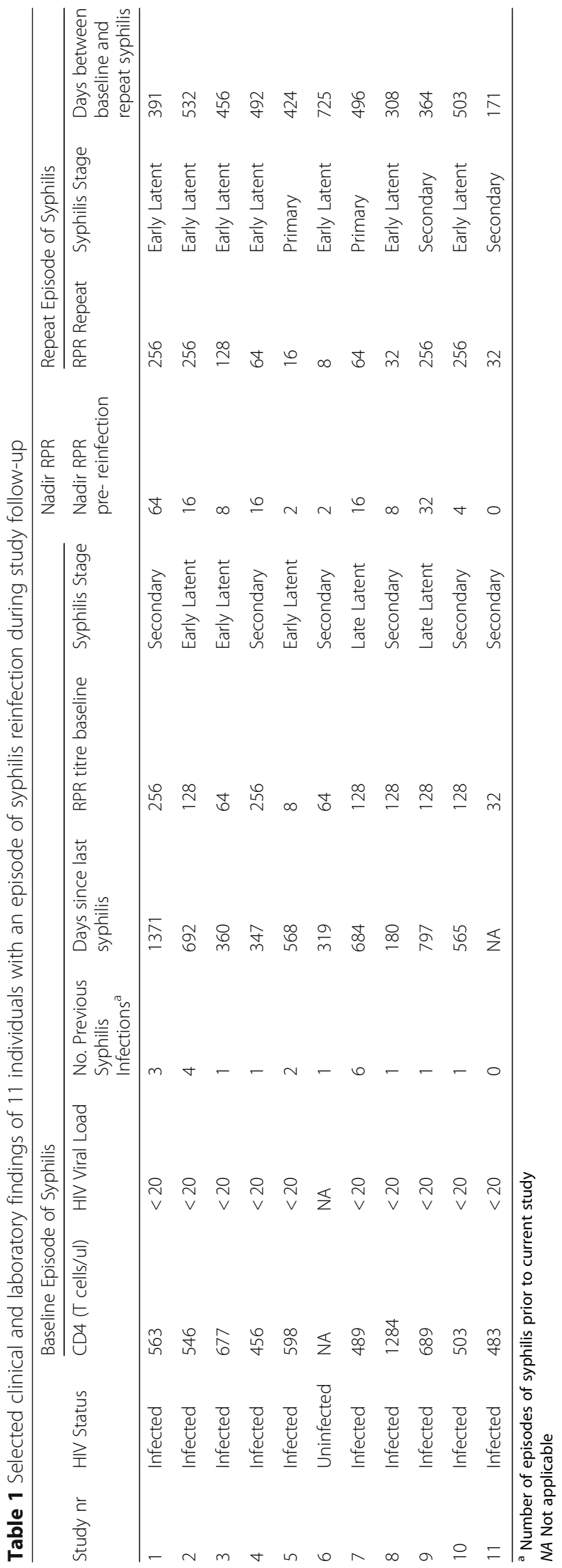


rash and an RPR titre rise to $1 / 32$. Of the 11 individuals, his baseline syphilis contained the highest or second highest levels of IL-10, IFN $\alpha$, CCL4 and IP-10 (Participant 11, Fig. 1). At the time of his reinfection his cytokine levels did not attain the levels of his initial infection (Fig. 1).

\section{Paired comparisons of cytokines}

Comparing to their baseline infections, paired analyses of the 11 individuals with reinfections during follow-up revealed that these reinfections had lower concentrations of IFNa $(0.8$ [IQR 0.8-0.8] vs. 12.2 [IQR 1.6-24.2], $P=0.004$ ) and CCL4 (0.9 [IQR $0.9-12.2$ vs. 17.5 [IQR 4.9-32.8], $P=0.022$ ];

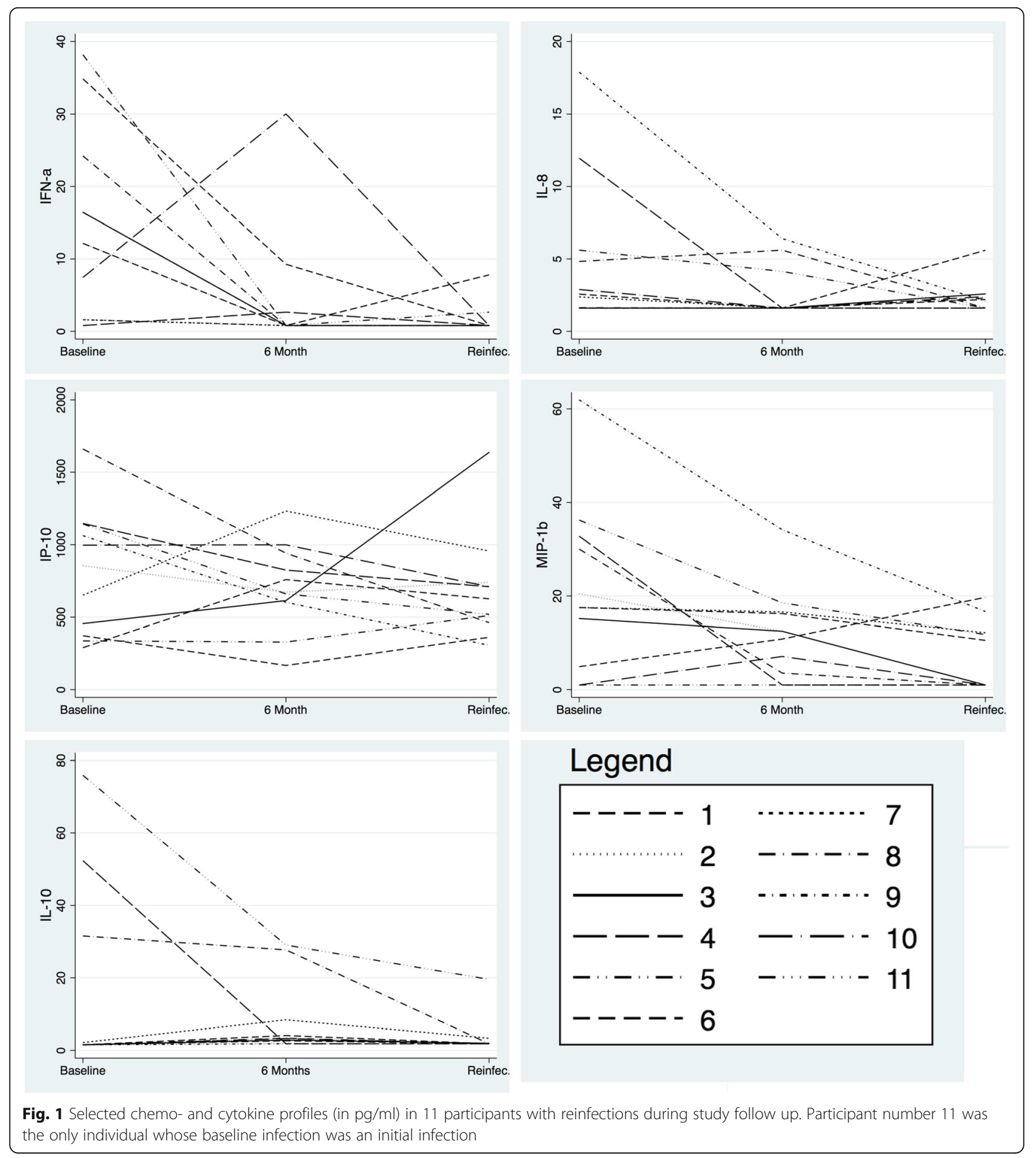


Table 2). These associations remained after excluding the individual whose baseline infection was his first (initial) episode of syphilis. In general, there was a decline in cyto- and chemokines between baseline and 6-month visit with little or no increase at the time of reinfection (Fig. 1).

\section{Correlation between number of prior episodes of syphilis and individual cyto/chemokines}

There was a weak negative association between IFN $\alpha$ and the number of episodes of syphilis $($ Rho $=-0.42$; $P=0.050$; Table 3).

\section{Discussion}

A diagnosis of syphilis in humans and animal models is typically characterized by elevations in both plasma pro-inflammatory (TNF $\alpha$, IFN $\gamma$, CCL4, IP-10) and anti-inflammatory cytokines (IL-10) [11-18]. This immunological profile however varies according to a number of parameters including stage of syphilis [4, 17-20], HIV-infection status [20] and the number of previous episodes of syphilis $[4,21,22]$. Our study is the first that we are aware of that has been able to assess the immune profile of the same individuals at the time of reinfection.

Our study is weakened by the small number included and the fact that only one of the 11 baseline episodes of syphilis was a confirmed initial episode of syphilis. Larger studies would be required to be able to conduct multivariable analyses to control for the role of confounders such as stage of syphilis, number of episodes of syphilis, time between episodes of syphilis and HIV status. With a sample size of only 11 we deemed the risk of type II errors too large to warrant conducting multivariable analyses. As a result we cannot exclude the possibility that our results are due to confounding. Nonetheless our results are commensurate with the thesis that repeat syphilis presents with an attenuated immune response. The individual with an initial syphilis at study inclusion had a more pronounced elevation in IL-10, IFN $\alpha$, CCL4 and IP-10 than the individuals with repeat syphilis. His second episode of syphilis was also clinically less dramatic and characterized by little or no elevation in plasma cytokines (compared to his 6-month levels). Furthermore, shortly after completing the 24-month follow-up period (and therefore not included in this study) this individual had a third episode of syphilis which was asymptomatic.

In the paired comparisons, plasma IFN $\alpha$ and CCL4 were lower at the time of the reinfection. The fact that repeat episodes had lower IFN $\alpha$ and CCL4 even after excluding the only individual whose baseline syphilis represented an initial infection suggests that each additional episode of syphilis results in a more attenuated immune response than the previous episode. As noted above, this finding is weakened by the fact that in 10 out of 11 individuals the baseline episode of syphilis was not the individual's first episode of syphilis. What we are comparing is earlier with later episodes of syphilis in the same individual. Our

Table 2 Plasma cytokine and chemokine concentrations in baseline, 6-month follow-up and repeat episodes of syphilis (pg/ml)

\begin{tabular}{|c|c|c|c|c|}
\hline & Baseline infection & 6 month & Repeat infection & $P$-Value \\
\hline IFNa & $12.1(1.6-24.2)$ & $0.8(0.8-2.6)$ & $0.8(0.8-0.8)$ & 0.004 \\
\hline IFNy & $1.5(1.5-8.9)$ & $1.7(1.5-10.3)$ & $1.54(1.5-10.6)$ & 0.714 \\
\hline IL1 $\beta$ & $1.5(1.5-1.5)$ & $1.57(1.5-1.5)$ & $1.57(1.5-1.5)$ & . \\
\hline IL12p40 & $1.0(1.0-1.0)$ & $1.0(1.0-1.0)$ & $1.0(1.0-1.0)$ & \\
\hline IL12p70 & $1.6(1.6-1.6)$ & $1.6(1.6-1.6)$ & $1.6(1.6-1.6)$ & . \\
\hline IP-10 & $855.4(372.7-1143.6)$ & $672.9(602.6-941.6)$ & $626.3(462.0-740.4)$ & 0.424 \\
\hline MCP-1 & $242.1(212.8-303.1)$ & $278.37(259.2-312.2)$ & 260.7 (225.8-294.0) & 0.533 \\
\hline CCL3 & $1.6(1.6-4.9)$ & $1.6(1.6-1.6)$ & $1.6(1.6-1.6)$ & 0.127 \\
\hline CCL4 & $17.5(4.9-32.8)$ & $12.4(3.5-16.6)$ & $0.9(0.9-12.1)$ & 0.022 \\
\hline IL-4 & $1.6(1.6-1.6)$ & $1.6(1.6-1.6)$ & $1.6(1.6-1.6)$ & \\
\hline IL-5 & $1.6(1.6-1.6)$ & $1.6(1.6-1.6)$ & $1.6(1.6-1.6)$ & . \\
\hline IL-6 & $1.3(1.3-1.3)$ & $1.3(1.3-1.3)$ & $1.3(1.3-1.3)$ & . \\
\hline IL-7 & $1.1(1.1-1.6)$ & $1.1(1.1-1.1)$ & $1.1(1.1-1.1)$ & . \\
\hline IL-8 & $2.5(1.6-5.6)$ & $1.6(1.6-4.1)$ & $2.1(1.6-2.4)$ & 0.265 \\
\hline IL-10 & $1.5(1.5-31.5)$ & $3.3(1.9-8.4)$ & $1.8(1.8-1.9)$ & 0.787 \\
\hline IL-17A & $1.5(1.5-1.5)$ & $1.5(1.5-1.5)$ & $1.5(1.5-1.5)$ & 0.489 \\
\hline RPR titre & $128(64-128)$ & $8(2-16)$ & $32(16-128)$ & 0.07 \\
\hline
\end{tabular}

All values are median and interquartile range

a P-value is for paired comparison of reinfections with baseline infections (Wilcoxon's signed rank test) 
Table 3 Spearman's correlations between plasma cytokine and chemokine concentrations at the time of syphilis diagnosis and number of prior diagnoses of syphilis (baseline and repeat infections combined, $N=22$ )

\begin{tabular}{lll}
\hline & Spearman's correlation & $P$-Value \\
\hline IFNa & -0.42 & 0.050 \\
IFNy & -0.07 & 0.761 \\
IL1 $\beta$ & - & - \\
IL12p40 & - & - \\
IL12p70 & 0.17 & 0.42 \\
IP-10 & -0.22 & 0.327 \\
MCP-1 & 0.141 & 0.531 \\
CCL3 & -0.09 & 0.697 \\
CCL4 & -0.27 & 0.222 \\
IL-4 & - & - \\
IL-5 & - & - \\
IL-6 & 0.17 & 0.428 \\
IL-7 & 0.14 & 0.532 \\
IL-8 & -0.31 & 0.156 \\
IL-10 & -0.04 & 0.84 \\
IL-17A & -0.28 & 0.202 \\
\hline
\end{tabular}

findings of lower plasma IFN $\alpha$ and CCL4 in later episodes of syphilis is commensurate with immunological and clinical findings from other studies. Previous studies have, for example, found that each subsequent diagnosis of syphilis is associated with a step-wise increase in peak RPR at the time of diagnosis, a faster decay in RPR but then a higher nadir RPR result following the episode of syphilis [4, 21, 22 ]. One of these studies found that the RPR result did not serorevert in any individuals with four or more episodes of syphilis [4]. A recent study has found a stepwise increase in the probability that syphilis presents asymptomatically with increasing numbers of episodes of syphilis [3]. These findings suggest that individuals with multiple episodes of syphilis may develop a degree of immunity to infection by $T$. pallidum [1] and that each episode of syphilis has an additive effect in the development of immunity. Further studies are required to assess if the attenuated immune response is correlated with a lower bacterial load and hence infectivity. This would be relevant for studies modeling syphilis spread and might provide clues of use for Treponema pallidum vaccine design.

In a similar vein, our study builds on the findings of other contemporary studies which suggest a need to rethink the syphilis staging system used in settings such as ours where repeat syphilis constitutes an increasing proportion of all syphilis infections [1]. In a study of individuals with four or more episodes of syphilis from our center, for example, we found a step-wise increase in the proportion diagnosed with latent (asymptomatic) syphilis with increasing numbers of episodes of syphilis [3]. Similarly, in this study we found that 7 of the 11 episodes of repeat syphilis were latent syphilis. This classification was based on the fact that these 7 episodes were asymptomatic. The traditional staging system of syphilis typically depicts syphilis as progressing from primary to secondary and then early and late latent syphilis [23]. A large proportion of our patients with repeat syphilis do not develop symptoms of syphilis and thus classified as latent syphilis. This is likely due to the attenuated immune response to repeat syphilis and not reflective of a longer duration of infection as would be the case in initial episodes of latent syphilis. Future studies could explore this issue further by assessing if there are clinical, immunological, pathogen and pathological differences between individuals with early and late latent syphilis diagnosed during a first compared to a fourth (or higher) episode of syphilis. Consideration could be given to classifying latent syphilis in individuals with multiple previous episodes of syphilis as latent-repeat syphilis [1].

Unfortunately we were unable to sequence $T$. pallidum DNA from any of the episodes of syphilis included. We were thus unable to establish genotypically if the repeat syphilis was due to reinfection or reactivation as has been done in other studies [24, 25]. Given the high rates of partner change, low rates of condom usage and high syphilis incidence in this cohort [4] it is however likely that most if not all the repeat episodes are due to reinfections. We cannot, however, exclude the possibility that the episodes of repeat syphilis are due to reactivation rather than reinfection. Relapse syphilis may be characterized by a different immunological response to reinfection syphilis and this may influence our results. This misclassification bias would however be expected to bias the results towards the null-hypothesis.

\section{Conclusions}

This study makes a small contribution to the growing evidence that the clinical and immunological presentation of repeat syphilis is more attenuated than initial syphilis. This is of considerable clinical and public health consequence. It is important that both clinicians and patients are aware of this as it provides extra motivation for periodic serological screening in persons who have had a diagnosis of syphilis. These individuals are not only at a considerably higher risk of syphilis than the general population, their subsequent syphilis is also more likely to be asymptomatic and serological screening may be the only way to diagnose this syphilis episode in a timeous fashion.

\section{Abbreviations}

CCL: Chemokine ligand; IFN: Interferon; IL: Interleukin; IQR: Interquartile

range; MIP: Macrophage inflammatory protein; MSM: Men who have sex with men; PCR: Polymerase chain reaction; RPR: Rapid plasma reagin; TNF: Tumour necrosis factor; USA: United States of America 


\section{Acknowledgements}

We would like to thank Said Abdellati and Vicky Cuylaerts for performing the cytokine assays.

\section{Funding}

This work was supported by the grants from the Flemish Government, Department of Economy, Science and Innovation, SOFI-B Grant to CK (Project ID 757003), http://www.fwo.be. The funders played no role in the design of the study or the collection, analysis or interpretation of the data.

\section{Availability of data and materials}

The datasets used and/or analyzed during the current study are available from the corresponding author on reasonable request.

\section{Authors' contributions}

$\mathrm{CK}, \mathrm{KO}, \mathrm{TC}$ and LK conceived the study. CK conducted the data analyses and wrote the first draft. CK, KO, TC and LK all contributed to the final version of the manuscript. All authors read and approved the final manuscript'

\section{Ethics approval and consent to participate}

The Institutional Review Board of the Institute of Tropical Medicine and the Ethics Committee of the University Hospital Antwerp approved this study (13/44/426). Individual written informed consent was obtained from each participant.

\section{Consent for publication}

Not applicable.

\section{Competing interests}

The authors declare that they have no competing interests.

\section{Publisher's Note}

Springer Nature remains neutral with regard to jurisdictional claims in published maps and institutional affiliations.

\section{Author details}

${ }^{1}$ HIV/STI Unit, Institute of Tropical Medicine, Antwerp, Belgium. ${ }^{2}$ Division of Infectious Diseases and HIV Medicine, University of Cape Town, Anzio Road, Observatory, Cape Town 7700, South Africa. ${ }^{3}$ HIV/STI Reference Laboratory, Institute of Tropical Medicine, Antwerp, Belgium. ${ }^{4}$ Immunology Unit, Institute of Tropical Medicine, Antwerp, Belgium. ${ }^{5}$ Department of Biomedical Sciences, University of Antwerp, Antwerp, Belgium.

Received: 1 March 2018 Accepted: 20 September 2018

Published online: 25 September 2018

\section{References}

1. Kenyon C, Lynen L, Florence E, Caluwaerts S, Vandenbruaene M, Apers L, Soentjens P, Van Esbroeck M, Bottieau E. Syphilis reinfections pose problems for syphilis diagnosis in Antwerp, Belgium - 1992 to 2012. Euro Surveill. 2014;19(45):20958.

2. Botham SJ, Ressler K-A, Maywood P, Hope KG, Bourne CP, Conaty SJ, Ferson MJ, Mayne DJ. Men who have sex with men, infectious syphilis and HIV coinfection in inner Sydney: results of enhanced surveillance. Sex Health. 2013:10(4):291-8.

3. Kenyon C, Osbak KK, Apers L. Repeat syphilis is more likely to be asymptomatic in HIV-infected individuals: a retrospective cohort analysis with important implications for screening. Open Forum Infect Dis. 2018; 5(6):ofy096.

4. Kenyon C, Tsoumanis A, Osbak K, Van Esbroeck M, Crucitti T, Kestens L. Repeat syphilis has a different immune response compared to initial syphilis: an analysis of biomarker kinetics in two cohorts. Sex Transm Infect. 2017; Epub ahead of print.

5. Courjon J, Hubiche T, Dupin N, Grange PA, Del Giudice P. Clinical aspects of syphilis reinfection in HIV-infected patients. Dermatology. 2015;230(4):302-7.

6. Brewer TH, Peterman TA, Newman DR, Schmitt K. Reinfections during the Florida syphilis epidemic, 2000-2008. Sex Transm Dis. 2011;38(1):12-7.

7. Kerani R, Lukehart S, Stenger M, Marra C, Pedersen R, Golden M. Is early latent syphilis more likely in patients with a prior syphilis infection? British Society for Sexual Health and HIV. London: Presentation at 18th international society for STD research; 2009. 28 June-1 July
8. Magnuson HJ, Thomas EW, Olansky S, Kaplan BI, De Mello L, Cutler JC Inoculation syphilis in human volunteers. Medicine (Baltimore). 1956;35(1):33-82.

9. Workowski KA, Berman SM, Centers for disease C, prevention. Sexually transmitted diseases treatment guidelines, 2010. Atlanta: Department of Health and Human Services, Centers for Disease Control and Prevention; 2010.

10. Chen CY, Chi KH, George RW, Cox DL, Srivastava A, Rui Silva M, Carneiro F, Lauwers GY, Ballard RC. Diagnosis of gastric syphilis by direct immunofluorescence staining and real-time PCR testing. J Clin Microbiol. 2006;44(9):3452-6.

11. Bernardeschi C, Grange PA, Janier M, Gressier L, Dion PL, Benhaddou N, Bianchi A, Lassau F, Avril MF, Batteux F, et al. Treponema pallidum induces systemic TH17 and TH1 cytokine responses. Eur J Dermatol. 2012;22(6):797-8.

12. Wicher V, Scarozza AM, Ramsingh Al, Wicher K. Cytokine gene expression in skin of susceptible Guinea-pig infected with Treponema pallidum. Immunology. 1998;95(2):242-7.

13. Salazar JC, Cruz AR, Pope CD, Valderrama L, Trujillo R, Saravia NG, Radolf JD. Treponema pallidum elicits innate and adaptive cellular immune responses in skin and blood during secondary syphilis: a flow-cytometric analysis. J Infect Dis. 2007;195(6):879-87.

14. Kojima N, Bristow CC, Maecker H, Rosenberg-Hasson Y, Leon SR, Vargas SK, Konda KA, Caceres CF, Klausner JD. Similarities in the markers of inflammation between men with syphilis and women with increased risk of HIV acquisition. Clin Infect Dis. 2016;62(2):265-6.

15. Cruz AR, Ramirez LG, Zuluaga AV, Pillay A, Abreu C, Valencia CA, La Vake C, Cervantes JL, Dunham-Ems S, Cartun R, et al. Immune evasion and recognition of the syphilis spirochete in blood and skin of secondary syphilis patients: two immunologically distinct compartments. PLoS Negl Trop Dis. 2012;6(7):e1717.

16. Knudsen A, Benfield T, Kofoed K. Cytokine expression during syphilis infection in HIV-1-infected individuals. Sex Transm Dis. 2009;36(5):300-4.

17. Podwinska J, Lusiak M, Zaba R, Bowszyc J. The pattern and level of cytokines secreted by Th1 and Th2 lymphocytes of syphilitic patients correlate to the progression of the disease. FEMS Immunol Med Microbiol. 2000;28(1):1-14.

18. Pastuszczak M, Gozdzialska A, Jakiela B, Obtulowicz A, Jaskiewicz J, Wojas-Pelc A. Robust pro-inflammatory immune response is associated with serological cure in patients with syphilis: an observational study. Sex Transm Infect. 2017; 93(1):11-14.

19. Fitzgerald TJ. The Th1/Th2-like switch in syphilitic infection: is it detrimental? Infect Immun. 1992;60(9):3475-9.

20. Kenyon C, Osbak KK, Crucitti T, Kestens L. The immunological response to syphilis differs by HIV status; a prospective observational cohort study. BMC Infect Dis. 2017;17(1):111.

21. Brown ST, Zaidi A, Larsen SA, Reynolds GH. Serological response to syphilis treatment. A new analysis of old data. JAMA. 1985;253(9):1296-9.

22. Romanowski B, Sutherland R, Fick GH, Mooney D, Love EJ. Serologic response to treatment of infectious syphilis. Ann Intern Med. 1991;114(12):1005-9.

23. Tramont E, In S, Mandell GL, Bennett JE, Dolin R. Principles and Practice of Infectious Diseases. 8th ed. Atlanta: Churchill Livingstone Inc; 2015.

24. Myint M, Bashiri H, Harrington RD, Marra CM. Relapse of secondary syphilis after benzathine penicillin G: molecular analysis. Sex Transm Dis. 2004;31(3):196-9.

25. Florindo C, Reigado V, Gomes JP, Azevedo J, Santo I, Borrego MJ. Molecular typing of treponema pallidum clinical strains from Lisbon, Portugal. J Clin Microbiol. 2008:46(11):3802-3.

Ready to submit your research? Choose BMC and benefit from:

- fast, convenient online submission

- thorough peer review by experienced researchers in your field

- rapid publication on acceptance

- support for research data, including large and complex data types

- gold Open Access which fosters wider collaboration and increased citations

- maximum visibility for your research: over $100 \mathrm{M}$ website views per year

At BMC, research is always in progress.

Learn more biomedcentral.com/submissions 